\title{
Dans la fabrique d'une archive théâtrale
}

Entretien mené par Aurore Desgranges

Julie Peghini et Nicolas Martin-Granel

\section{(2) OpenEdition}

1 Journals

Édition électronique

URL : https://journals.openedition.org/coma/4553

DOI : $10.4000 /$ coma.4553

ISSN : 2275-1742

Éditeur

Institut des textes \& manuscrits modernes (ITEM)

Référence électronique

Julie Peghini et Nicolas Martin-Granel, « Dans la fabrique d'une archive théâtrale », Continents manuscrits [En ligne], 13 | 2019, mis en ligne le 21 novembre 2019, consulté le 14 janvier 2023. URL http://journals.openedition.org/coma/4553 ; DOI : https://doi.org/10.4000/coma.4553

Ce document a été généré automatiquement le 14 janvier 2023.

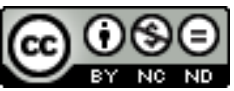

Creative Commons - Attribution - Pas d'Utilisation Commerciale - Pas de Modification 4.0 International - CC BY-NC-ND 4.0

https://creativecommons.org/licenses/by-nc-nd/4.0/ 


\title{
Dans la fabrique d'une archive théâtrale
}

\author{
Entretien mené par Aurore Desgranges
}

Julie Peghini et Nicolas Martin-Granel

1 Dans cet entretien mené par Aurore Desgranges le 23 mai 2019, Nicolas Martin-Granel et Julie Peghini posent un regard rétrospectif sur leur collaboration et reviennent sur les différentes étapes théoriques et méthodologiques de leur questionnement sur les archives théâtrales de Sony Labou Tansi. Un ouvrage sur l'ensemble de son œuvre de dramaturge est en effet en préparation au sein de l'équipe Manuscrits francophones et marquera l'aboutissement d'un long cheminement intellectuel.

Fig. 1: «Pourquoi le théâtre ?»

Feuillet manuscrit non daté, voir fac-similé et transcription dans Sony Labou Tansi, La Chair et I'Idée, Besançon, Les solitaires intempestifs, 2015, p. 149-151, repris en partie dans Sony Labou Tansi, Encre, sueur, salive et sang, Paris, Le Seuil, 2015, p. 93-94. 


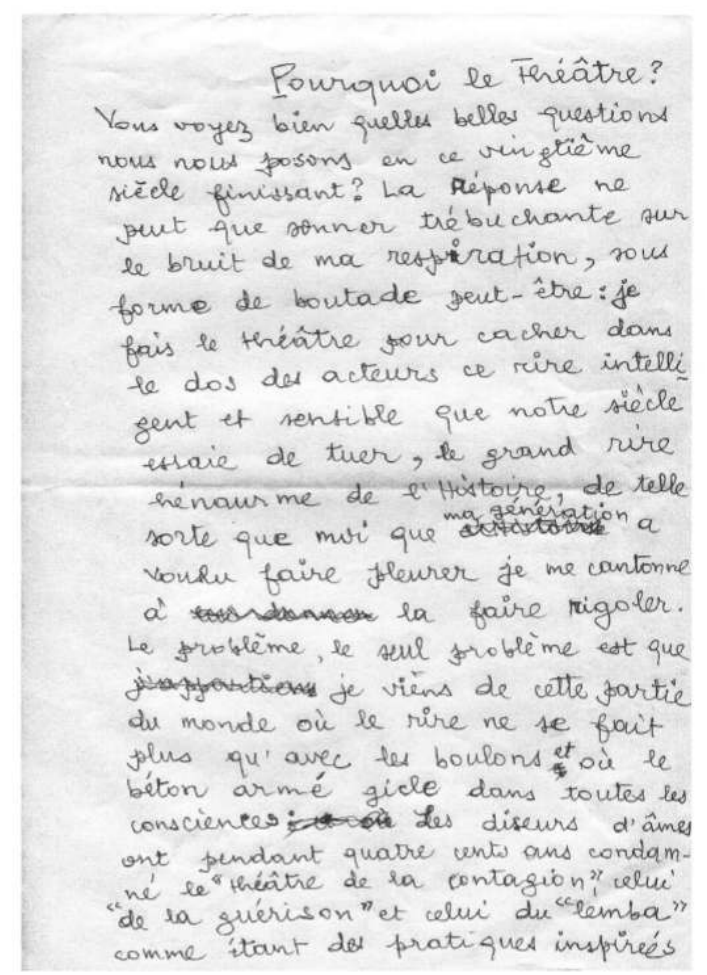

2 Après la mort de Sony Labou Tansi en 1995 à Brazzaville, Nicolas Martin-Granel a coorganisé plusieurs inventaires de ce qui est devenu le fonds Sony Labou Tansi ${ }^{1}$ : un premier travail de localisation et d'inventaire des manuscrits et livres dans la « chambre-bureau » de l'auteur avec les proches à Brazzaville (Victor MBila Mpassi et Apollinaire Singou-Basseha). Cinq ans après la guerre civile de 1997-1998, il retourne à Brazzaville avec Greta Rodriguez-Antoniotti pour un inventaire des manuscrits « survivants » et découvre des manuscrits inconnus. En 2011, il participe à une mission spécifique de numérisation dans le cadre de l'équipe Manuscrits francophones. L'ensemble de ces recherches a permis la publication de plusieurs inédits entre 2005 et 2015 (les correspondances de Sony Labou Tansi avec Françoise Ligier et José Pivin, deux recueils de poèmes, le roman Machin la Hernie ${ }^{2}$, ses essais ${ }^{3}$, une édition génétique des poèmes ${ }^{4}$ et La Chair et l'Idée ${ }^{5}$ dont il sera question dans cet entretien). Le constat de la dispersion de l'œuvre de Sony Labou Tansi, aussi bien dans les archives des instances de consécration de la francophonie (le dépôt de Monique Blin au pôle francophone de la $\mathrm{BnF}$, archives du concours théâtral interafricain) qu'auprès de ses amis et compagnons de route (les anciens de la troupe du Rocado Zulu Théâtre qu'il co-fonde à Brazzaville avec Nicolas Bissi, les metteurs en scène, auteurs et éditeurs avec lesquels il a travaillé comme Guy Lenoir, Caya Makhélé, etc.), donne lieu à la création du fonds Sony Labou Tansi à la Bibliothèque francophone multimédia de Limoges.

3 C'est autour de leur intérêt pour l'activité théâtrale de Sony Labou Tansi que Julie Peghini et Nicolas Martin-Granel entament leur collaboration scientifique et exploiteront peu à peu ces pistes tout en en découvrant de nouvelles. En septembre 2013, dans le cadre du projet «Les Testaments de Sony Labou Tansi », soutenu par le Labex Arts H2H, Julie Peghini co-organise avec Jean-François Dusigne un stage de direction d'acteurs animé par Jean-Damien Barbin et Dieudonné Niangouna autour de la poésie de Sony Labou Tansi. Un film co-réalisé par Julie Peghini et Laëtitia Biaggi est consacré à ce travail : SLT : lieu de C(l)asse (2015). En clôture du projet, Jean- 
Damien Barbin, Julie Peghini et Nicolas Martin-Granel coordonnent un colloque : "Sony Labou Tansi en scène(s): une expérience théâtrale du monde » en novembre 2013 au Conservatoire national supérieur d'art dramatique de Paris. C'est l'occasion de restituer des travaux d'élèves du conservatoire mais également de convoquer des paroles plurielles venant témoigner de l'activité théâtrale de Sony Labou Tansi. Deux ans plus tard, Julie Peghini et Nicolas Martin-Granel publient Sony Labou Tansi en scène(s), La Chair et l'Idée $e^{6}$, ouvrage qui retrace au fil de sa correspondance sa trajectoire nomade et cosmopolite entre le Congo et la France, offre à la connaissance du lecteur des textes inédits (le poème La Troisième France, la pièce de théâtre La Gueule de rechange) et relaie la parole de ses multiples compagnons de route.

4 Cet ouvrage est le fruit d'une recherche décloisonnée sur le geste théatral de Sony Labou Tansi et s'appuyant sur une exploration minutieuse du fonds. Julie Peghini et Nicolas Martin-Granel distinguent en effet différentes "scènes " dans l'expérience théâtrale sonyenne :

- en 1973-1974, le voyage en France sur l'invitation de José Pivin et Françoise Ligier ${ }^{7}$ et le maintien d'une correspondance ;

- en 1980-1984, l'expérience du Rocado Zulu Théâtre qui amène différents metteurs en scène à travailler avec lui au Congo, au festival des Francophonies en Limousin, au TILF (Théâtre international de langue française) et lors de la tournée du BBKB (Bordeaux-BrazavilleKinshasa-Bangui);

- en 2013-2014, le stage de direction d'acteurs au conservatoire qui met en évidence la théâtralité de son écriture poétique et la nécessité de penser et de perpétuer son legs artistique.

L'ensemble de ces travaux s'inscrit ainsi dans une volonté de nourrir une mémoire vive autour de l'œuvre de l'auteur. C'est ainsi que la recherche scientifique fait écho à de nombreux projets artistiques :

- en 2016, Jean-Paul Delore met en scène Machin la Hernie avec Dieudonné Niangouna ;

- en 2016, Étienne Minoungou et Patrick Janvier mettent en scène Si nous voulons vivre à partir des essais de Sony8.

Depuis ces événements Julie Peghini et Nicolas Martin-Granel ont fait de nombreux allers-retours à Brazzaville. Ils travaillent sans relâche à la réunion de ce fonds dispersé auprès d'amis et comédiens, suivent de près l'héritage de son action, notamment via le festival Mantsina-sur-scène ${ }^{9}$, et réfléchissent à ce qui constituera le cœur du prochain ouvrage sur son théâtre avec les autres membres du groupe "Sony Labou Tansi»: Amélie Thérésine, Céline Gahungu, Sonia Le Moigne Euzenot, Alice Desquilbet, Suzanne Nzouzi, Patrice Yengo ${ }^{10}$.

6 C'est sur leur travail de fabrique d'une archive théâtrale en flux continu qu'ils ont accepté de répondre à mes questions.

\section{Le manuscrit entre archive, texte et performance}

AD : À travers l'ensemble de vos travaux et plus spécifiquement dans votre travail sur La Chair et l'Idée avez-vous précisé votre regard sur ce pan moins étudié de l'histoire de l'œuvre de Sony?

NMG : Je dirais qu'il s'agit d'une réflexion plus anthropologique qu'historique parce qu'on retrouvait par exemple dans la direction d'acteurs de Sony des points communs avec ses écrits. C'est cela que l'on recherchait. Je me souviens d'un colloque 
de l'association APELA ${ }^{11}$ en 2013 qui se passait à Bordeaux sur le thème «archive, texte, performance » : j'avais improvisé un petit peu là-dessus, comme je sortais tout bouillant de l'atelier ${ }^{12}$ (...) Dans le triangle archive-texte-performance, on met le manuscrit au milieu parce que c'est à la fois un texte, une archive et une performance. Et je pense que c'est parce que j'ai le souvenir de Dido ${ }^{13}$ engueulant ses acteurs et actrices, qui disait : «Soyez des faisant, ne cherchez pas à savoir ce que ça veut dire, à revenir en arrière, à expliquer, etc.». On est donc en plein dans la question de la poétique, du « poiein ».

JP : Et puis il disait : "Arrêtez de questionner : pourquoi on fait ça, pourquoi vous voulez nous faire faire ça, où voulez-vous nous emmener?». Certains élèves se questionnaient sur le statut de cette parole qui venait souvent très tard après le corps, après une mise en danger et ce que tu dis, Nicolas, ce triangle, me semble très important, y compris dans ce que l'on a fait par rapport à ce livre, c'est-à-dire vraiment le manuscrit, le théâtre, la performance.

NMG : Oui bien sûr, on a les trois dimensions en même temps, qui forment une sorte de cercle, vicieux ou vertueux; de fait, le manuscrit autographe s'inscrit au centre en ce qu'il représente, sans solution de continuité, à la fois la trace d'une performance d'écriture, d'un texte en cours d'élaboration et d'une archive au sens pleinement étymologique d'archè : il a vocation à commencer/commander; en somme, ce que Sony appelait «le quatrième côté du triangle ", un titre mana qu'il a donné à pas moins de sept de ses manuscrits ${ }^{14}$. Cela fait bouger les catégories qu'on peut avoir en littérature, en critique littéraire et même en anthropologie du théâtre.

JP : Par ailleurs, ce travail, nous avons mis deux ans à le faire, c'est-à-dire qu'à l'issue $\mathrm{du}$ colloque, nous avions une matière très riche et plurielle, des interventions, des échanges, des archives, des spectacles et nous nous référions dans un premier temps à la revue Équateur ${ }^{15}$. Ce qui est intéressant, c'est que Jean-Damien Barbin avait l'idée de prolonger ce coup de maître en lui donnant une suite. 
Fig. 2 : Première de couverture de la revue Équateur, n 1, dirigée par Caya Makhélé en 1985 à Paris

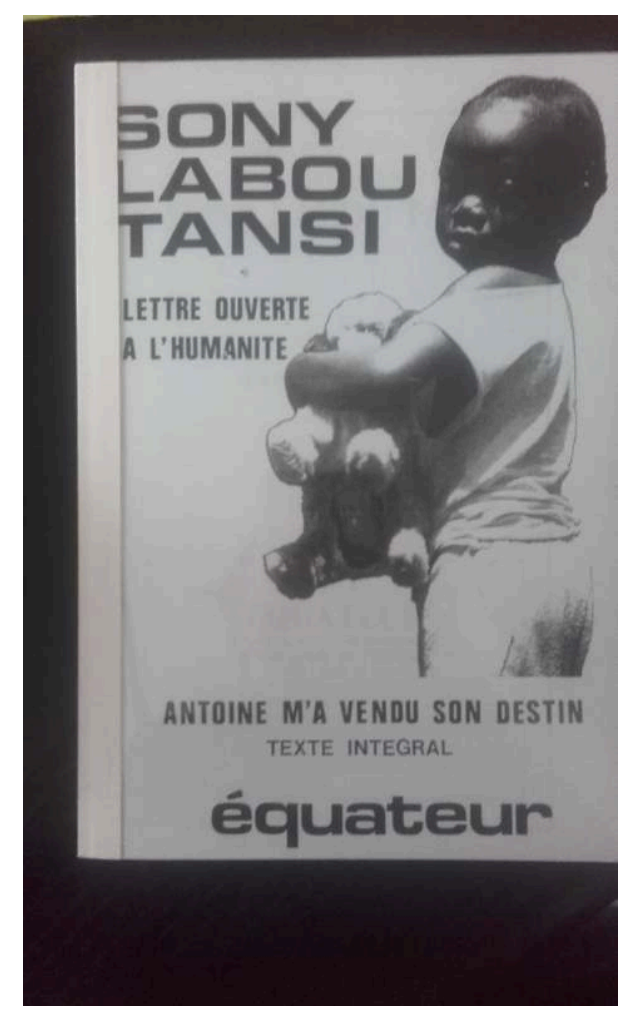

NMG: Ça, c'était la marotte de Jean-Damien, on ne pouvait pas faire ce deuxième numéro, on n'est pas dans les mêmes conditions historiques, on n'a plus Sony sous la main pour écrire et on ne peut pas écrire à sa place...

JP : En revanche, il y a quelque chose de très vivant dans cette revue qu'on a essayé de garder dans La Chair et l'Idée.

$A D$ : Il y a une inspiration...

JP : Oui... nous nous sommes dit : il y a autant de textes de Sony que de textes d'autres auteurs, il y a un devenir de cette œuvre qui était déjà dans la performance. Il y a un aspect théâtral de cette œuvre qui était dans ses manuscrits mais au sens très large, $y$ compris dans la poésie. Et l'on ne va pas séparer le théâtre de la poésie, on ne va pas mettre les mots de Sony à l'écart d'autres mots qui les performent aujourd'hui ou qui les prolongent. On va faire entendre la parole de ceux qui ont vécu l'expérience au Conservatoire, comme celle de ses compagnons de route et on va restituer la voix de ceux qui ont parlé pendant les rencontres, en étant au plus près de l'oralité, par une transcription scrupuleuse de ce qui avait été dit par chacun, donc il y a tous ces enjeux-là.

NMG : J'ajouterai que Sony est aussi un théoricien du théâtre...

JP: C'est ce qu'on a découvert, et on a fait une vraie recherche là-dessus...

NMG : Parce que dans la correspondance il y a des textes un peu théoriques qui ne sont pas sous la forme d'un traité. Il y a des paroles comme ça, un peu à la Artaud, qui vont à contre-courant. Il faut dire également qu'on a enlevé la partie universitaire en se disant qu'on la publierait dans les $E L A^{16} \ldots$ 
JP : ... En se disant que cela ne faisait pas partie de ce triangle-là, dont on est en train de te parler, et que dans ce triangle-là, il fallait rester au cœur des textes théoriques et des textes de théâtre ou de poésie, comme La Gueule de rechange et La Troisième France.

NMG : Moi je reste très «meschonnicien ", adepte de la poétique du rythme d'Henri Meschonnic. On voulait accompagner «le sujet de l'écriture» Sony et non pas le prendre comme objet, ce qu'aurait fait une étude universitaire, on voulait procéder d'une autre manière, par touches, par petites touches. [...]

JP : Puis il y a dans l'ouvrage une certaine logique qui se déroule jusqu'au devenir de l'œuvre : il s'agit de commencer par les inédits, le colloque, puis les écrits théoriques de Sony sur le théâtre. Ensuite, ses compagnons de route, avec cette oralité dont je parlais, et enfin le legs, c'est-à-dire tous les textes ou performances qui nous parlent aujourd'hui de Sony et le transmettent. [...]

\section{Archives institutionnelles, archives intimes}

AD : Dans La Chair et l'ldée vous avez réuni les témoignages des metteurs en scène qui ont travaillé avec lui. Au fond, on peut retrouver à travers ces archives qui sont privées, intimes même, l'histoire institutionnelle de la francophonie. Est-ce que c'était quelque chose qui vous a intéressés?

JP : Non, nous avons vraiment demandé à ses compagnons de route des lettres de Sony dont ils étaient destinataires. C'est ainsi que nous avons sollicité Monique Blin ${ }^{17}$, Guy Lenoir, Michel Rostain, Gabriel Garran, Alain Timar, Daniel Mesguich, ou encore repris des échanges avec Jean-Pierre Klein...

NMG : Pour répondre à ta question directement : non justement, on ne voulait pas traiter de la question de la francophonie, de Limoges...

$A D$ : En tout cas, cela donne des informations sur le contexte de consécration...

NMG : C'était toutefois décentré par rapport à Limoges... C'était même l'intention de montrer qu'il y a du théâtre de Sony sans Limoges... Ce que nous voulions montrer, c'est qu'il n'y avait pas forcément une préséance de la part de Limoges. Le théâtre avait déjà commencé dès les années soixante-dix, avec les «dramatisations » que Sony donnait à jouer à ses collégiens... Toute cette partie immergée qu'on n'a pas encore publiée, les pièces de théâtre envoyées au Concours théâtral interafricain ... Limoges, c'est en 1985, c'est le dernier épisode. C'est pour cela qu'on a mis La Troisième France qui date de 1973, en guise de « scène primitive ».

JP : C'est la première "tropicalité », enfin, la première scène, pourrait-on dire : c'est l'idée qu'il est arrivé par là et que ça lui a fait écrire La Gueule de rechange et cela nous intéressait de redemander aux personnes concernées leur correspondance avec Sony pour savoir aussi comment elles travaillaient sur place. La centralité, elle était beaucoup pour nous dans ces relations-là, à Brazzaville justement. Que sont ces carnets de route? Qu'est-ce qui peut nous permettre de comprendre ce qui se passe avant la pièce à Limoges? Et qu'est-ce que ce processus ? Cela complète donc un point de vue sur le pourquoi de ces collaborations, et c'est une première enquête pour nous là-dessus. Nous avions justement invité au colloque ses compagnons de 
route congolais, notamment ceux du Rocado Zulu Théatre, à savoir Nicolas Bissi, Michel Matondo, Georges Mboussi, Victor Mbila Mpassi...

NMG : On a quelques traces, mais on n'a pas la prétention d'en avoir fait une histoire...

JP : Cela donne des informations plus complètes (...) et c'était une démarche rigoureuse, c'est-à-dire qu'on est vraiment allé demander à chacune de ces personnes leurs correspondances, leurs archives, ce qu'elles avaient pu conserver. Je me souviens très bien de cette discussion et de notre travail : c'est de se dire que la correspondance est très importante, il y avait eu celle à Françoise Ligier et José Pivin publiée dans le coffret en 2005.

NMG : Oui, parce que c'est le premier geste théâtral. (...)

JP : Et les recherches se sont enchainées ainsi, nous sommes allés voir Garran ${ }^{18}$, et voilà nos travaux ont pris forme. C'était important pour nous, après l'édition des textes inédits, de resituer le geste théâtral bien en amont de Limoges. Cela a montré à quel point cette correspondance nourrit l'acte de compagnonnage et donc l'acte théâtral pour Sony. D'ailleurs Nicolas vient de retrouver une carte postale que Sony a écrit à Monique Blin... où il lui dit ceci :

Monique, la machine repart, notre arbre poussera et sera comme ce baobab. J'ai écrit vingt pages ce dimanche à Dakar, une pièce comme je t'en parlais, pense à quelqu'un qui viendrait comme Vial pour collaborer à la mise en scène, ça me sort d'une lecture trop étroite de moi-même.

Je pense que c'est une phrase importante par rapport à notre démarche...

NMG: En particulier, comme Devésa ${ }^{19}$ le dit dans sa thèse, le festival des Francophonies a imposé son carcan. Là, il y a peut-être eu une servitude, fût-elle volontaire.

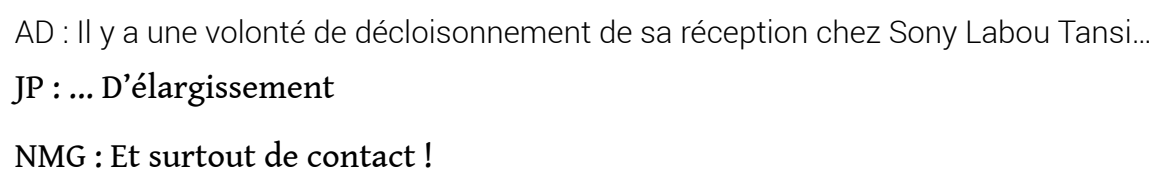

JP : C'est un poète du contact. Xavier Garnier en parle beaucoup dans son livre Sony Labou Tansi, une écriture de la décomposition impériale ${ }^{20}$, c'est très important. Dans la correspondance, c'est cet aspect du contact qui nous intéressait vraiment.

NMG: Cela s'accorde bien avec la poétique du fleuve, c'est toujours vers l'élargissement qu'il incline, alors que les éditeurs, eux, vont dans le sens du rétrécissement parce qu'ils doivent cocher des cases, mais là on voit bien que pour lui ce qui importe, c'est un élargissement...

JP : Et nous, avec ce type de démarche, il s'agissait d'élargir la réception de son théâtre. Je finis de lire la carte postale de Sony à Monique Blin :

Travaillons comme cela pendant un temps et puis nous changerons de cap si on veut, je cherche un titre à cette histoire des machinations provisoires...

Et c'est intéressant son changement de cap, c'est-à-dire qu'il n'y a rien de fixé, il n'y a pas de carte de route sur le fleuve, il y a juste cette idée de l'élargissement, ça ne lui est pas qu'imposé. Parler de la francophonie ne constituait pas l'intérêt majeur parce que le contact nous semble plus universel dans sa poétique. 
$A D$ : Et donc, aujourd'hui, vous avez aussi activé ou réactivé un certain nombre de réseaux. Comment cela s'organise-t-il maintenant pour travailler sur le théâtre de Sony ? II y a aussi l'enjeu d'une enquête qui est multisite parce que vous allez aussi au Congo régulièrement...

NMG : Le chantier actuel... Jusque-là, nous menions une démarche un peu fantaisiste. Là, il faut que nous soyons un peu plus dans les clous scientifiques, il y a une équipe plus étoffée, nous allons produire un travail plus systématique. Du point de vue de l'enquête, c'est sûr que nous commençons à accumuler beaucoup de choses... Des heures d'entretiens, mais aussi des versions scéniques annotées par les comédiens, des témoignages écrits, des photos de représentation, des coupures de critique théâtrale dans la presse locale, des pièces inédites écrites par des compagnons de Sony comme Nicolas Bissi ou Victor Louya.

$A D$ : Les gens s'adressent à vous directement quand ils ont quelque chose, donc vous êtes identifiés?

NMG : Bien sûr, ce sont des gens que je fréquentais déjà à Brazzaville au début des années 1990, surtout des anciens comédiens ou musiciens du Rocado mais aussi des amis de Sony, comme le journaliste Alphonse Nzanga Konga, qui se retrouvaient dans l'ANEC ${ }^{21}$.

JP : La publication de La Chair et l'Idée a été pour moi, qui n'ai pas l'histoire de Nicolas avec Brazzaville et qui n'ai pas connu Sony, un point de départ, qui a posé des bases pour continuer l'enquête...

NMG : On a peut-être acquis une légitimité là-dessus, c'est un livre qui sert de carte de visite. Il est vrai que l'on travaille davantage maintenant sur le terrain à Brazzaville, je ne sais pas, je pense qu'on a épuisé le terrain ici, il y a peut-être un moment, où il faut revenir sur les anciens du Rocado, creuser dans le champ où Sony a puisé sa sève, une sève qu'on découvre collective... Ainsi Nicolas Bissi nous a-t-il indiqué tel passage de sa pièce Sur la tombe de ma mère, que Sony avait tenu à ajouter, un passage qui porte sur les rapports « nord-sourd »...

JP : Il y a surtout une urgence à Brazzaville. Là, un des artistes qu'on a beaucoup interrogé l'an dernier, lors d'un séjour prolongé, est mort... Nous ne l'avons pas retrouvé cette année, il nous a donné des manuscrits, il nous a beaucoup parlé, il s'appelle Clément Batsimba, un des anciens comédiens du Rocado. Nous avons vraiment conscience qu'à Brazzaville, il y a urgence à recueillir des archives détenues par ses compagnons de route qui vieillissent...

NMG : Tous les ans, on retrouve des petites choses... on fait un peu les fourmis...

JP : Il nous faut maintenant travailler beaucoup plus systématiquement sur les pièces de théâtre. Nous reprenons chaque pièce et chacun de nous fait la genèse de ces pièces, nous sommes vraiment dans la lecture des textes de manière très précise pour comparer les différentes versions, pour remettre à jour certains avant-textes ou certains documents qui sont des archives de mise en scène. Outre les tapuscrits de répétition, nous nous efforçons de retrouver des images, des photos ou des vidéos, à l'INA par exemple, de retrouver des manières de pouvoir parler aujourd'hui de ce qu'étaient ces mises en scène aussi.

NMG : Pour le moment on est en chantier, parce qu'on ne sait pas ce qu'on pourra y mettre, parce qu'on est limité par le format papier.

JP : Le théâtre de Sony est énorme et il y a toutes ces pièces... 
AD : Pour autant, cela ne vous limite pas dans la collecte. J'ai l'impression que votre archive déborde...

JP : En fait, je pense que notre archive va continuer à déborder parce que nous avions déjà une telle base et que nous sommes encore plus de fourmis à travailler. Cela va nous permettre de densifier cette archive-là. C'est très important parce que nous avons entamé un travail plus systématique sur chaque pièce, à travers les versions publiées ou non, la correspondance, les photos, les archives sonores, les archives visuelles...

NMG : En génétique pour l'édition verticale ou horizontale. Là c'était les petits coups de sonde, comme ça, à sauts et à gambades... Mais il va falloir aussi se limiter de manière systématique parce qu'on n'aura pas assez de pages pour y arriver et puis on a des trous. Il y a des pièces dont on n'a pas de traces, qui étaient purement orales, générées à partir d'une improvisation musicale et chorégraphique... Je pense notamment à deux pièces tardives, dont j'ai pu voir la création à Brazzaville au début des années 1990, Franco l'âge de Dieu ${ }^{22}$ et Confession nationale ${ }^{23}$.

Fig. 3 : Recto et verso d'une lettre au conseiller culturel au sujet de la pièce Confession nationale, répertoriée dans le premier inventaire réalisé en 1996 et publié en 2003 dans Études Littéraires Africaines, $\mathrm{n}^{\circ} 15, \mathrm{p} .40$, dans ses deux versions, mss et tps.
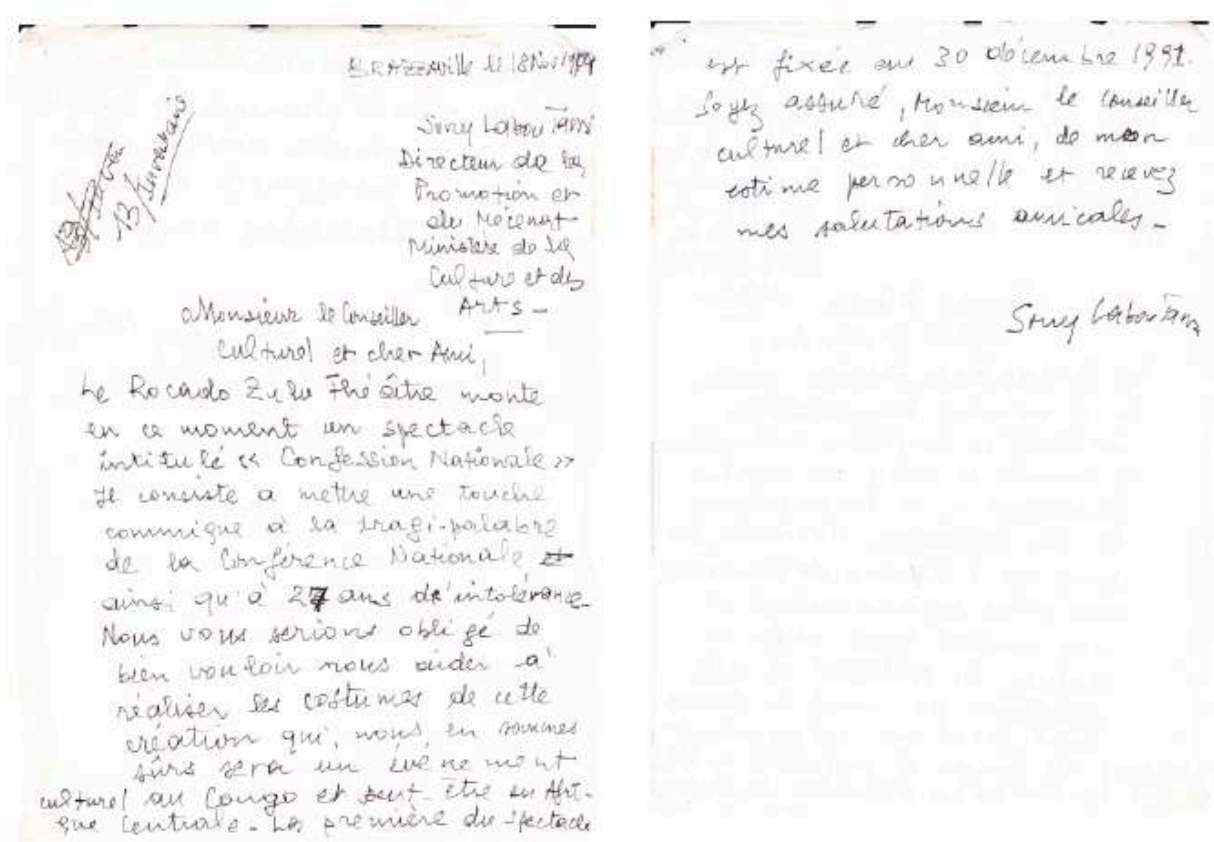
Fig. 4 : Devis estimatif du décor et costumes de la pièce Confession nationale

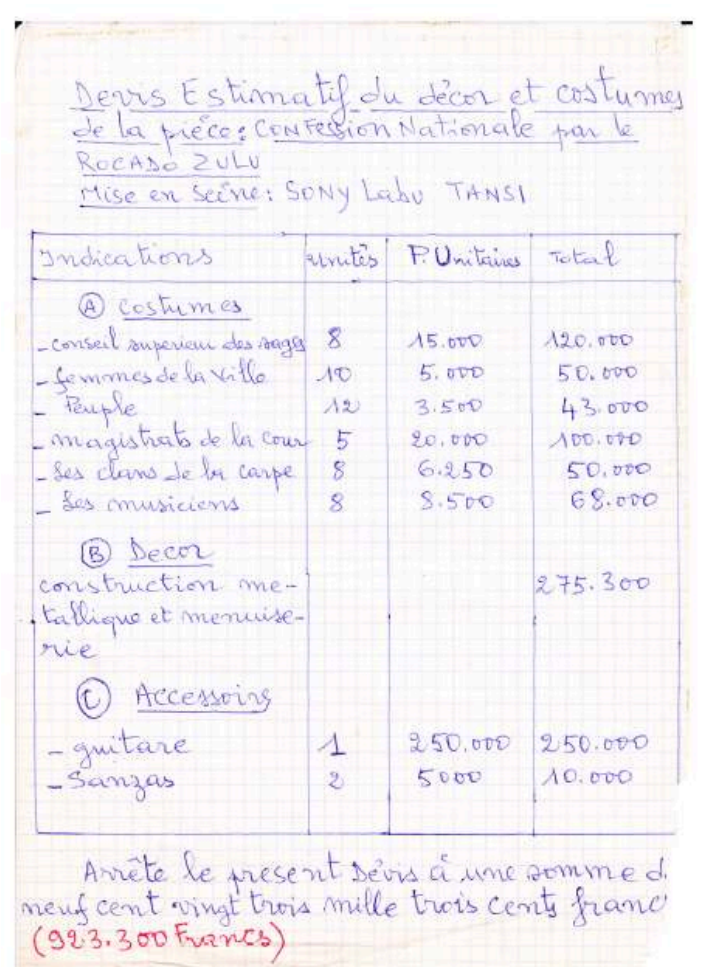

Fig. 5 : Listes d'invités internationaux (recto) et locaux (verso) pour la pièce Confession nationale
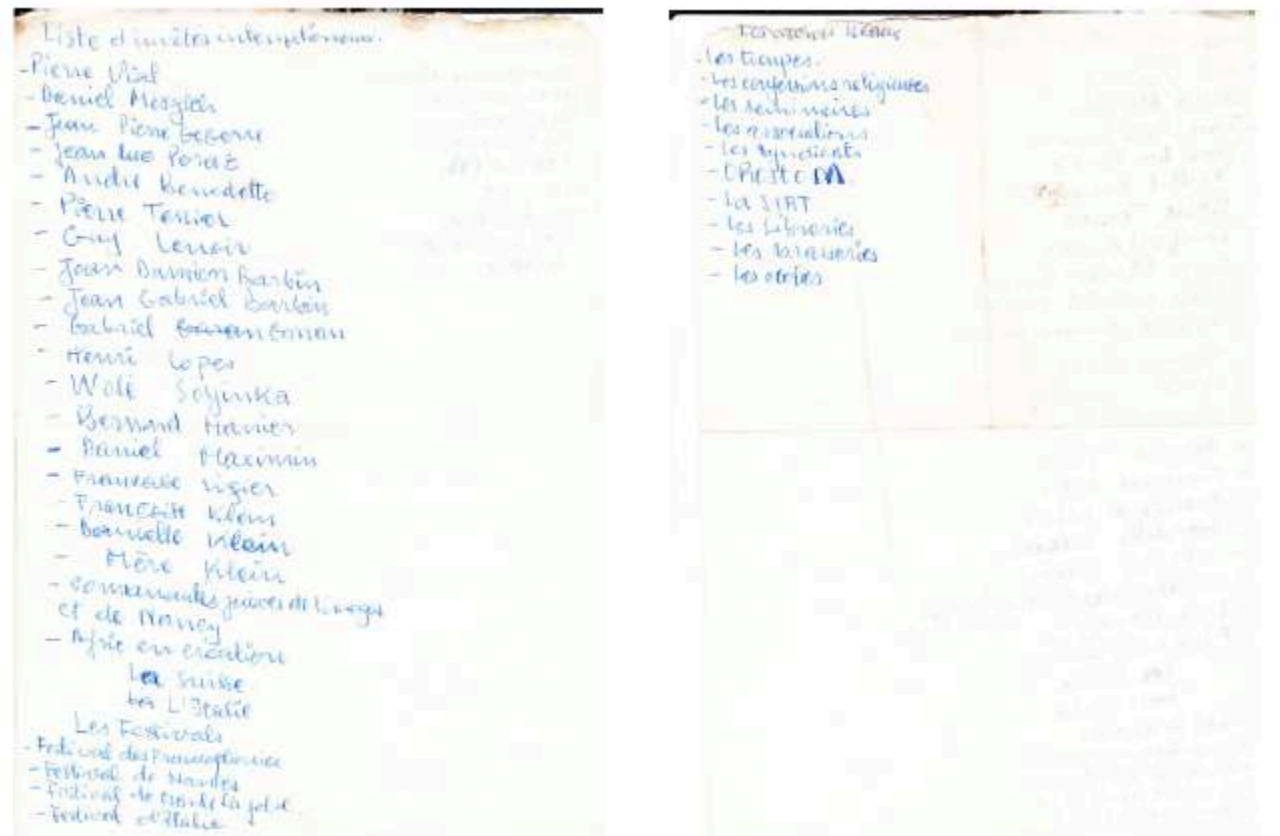
Fig. 6 : Liste des membres du Rocado Zulu Théâtre sur agenda (recto et verso)
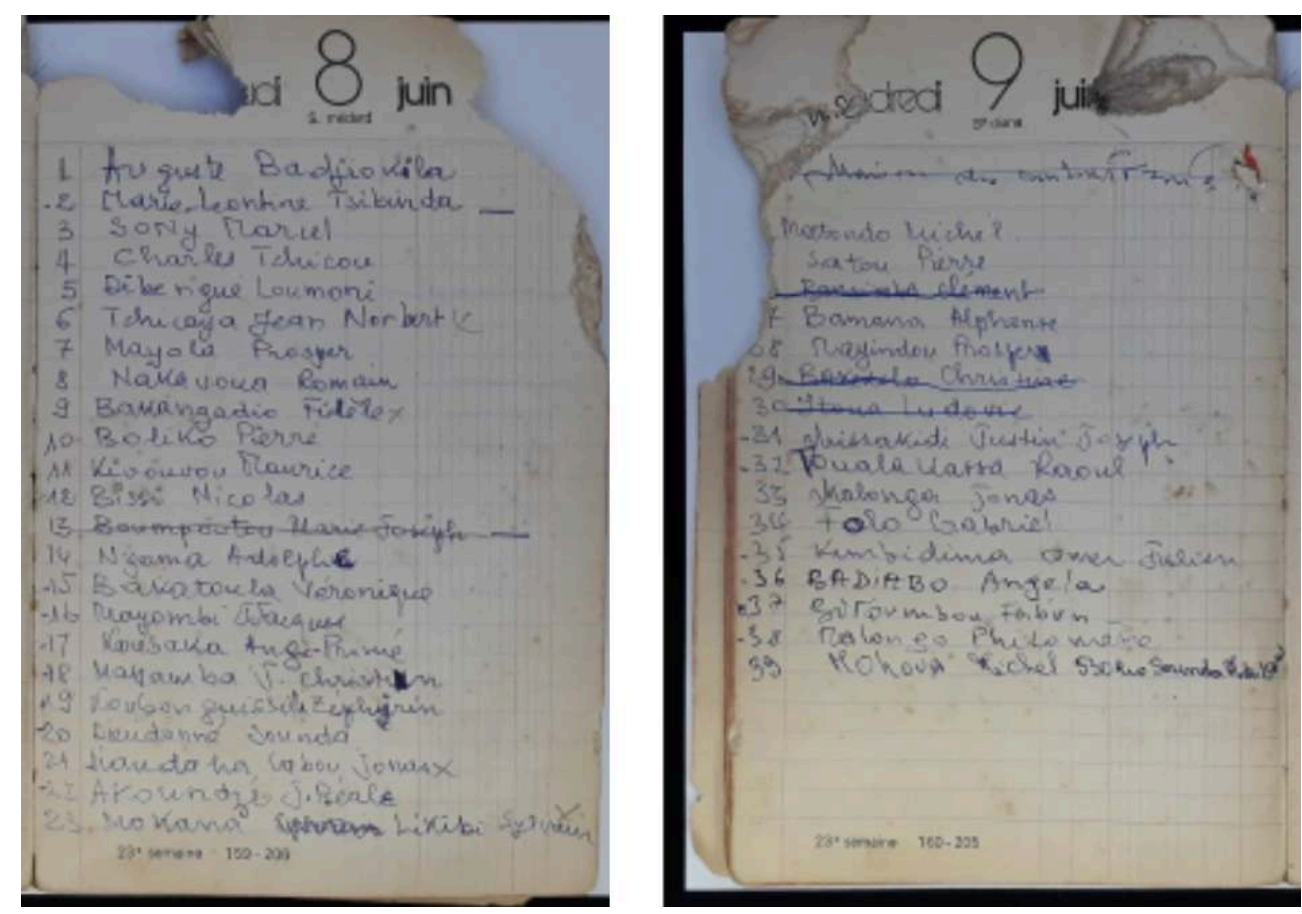

JP : Des traces orales qu'on repeuple progressivement [...] Par hasard, nous étions chez Nicolas Bissi ${ }^{24}$. Je n'avais pas cessé de photographier durant la journée. Quand je suis sortie, j'avais 1500 photos. Dans ces cas-là, tu ne regardes pas ce que tu photographies et nous avons ainsi trouvé un texte de théâtre que nous n'avions pas, des fragments de la pièce Kimpa vita ${ }^{25}$, une pièce qui n'a jamais été publiée. De même pour Franco, loge de $\mathrm{Dieu}^{26}$, nous commençons à avoir de plus en plus d'informations, c'est-à-dire que nous essayons de voir comment combler les manques par des traces et donc c'est important parce que nous retrouvons vraiment des documents précieux...

\section{Un pont vers l'histoire du théâtre à Brazzaville ?}

$A D$ : Vous parvenez à compléter des informations contextuelles sur l'histoire du théâtre à Brazzaville?

JP : Absolument, nous sommes allés faire des entretiens avec des metteurs en scène encore en vie à Brazzaville qui ne sont pas seulement les compagnons de route les plus connus. Nous avons vraiment élargi la base des entretiens...

NMG : Il faudra peut-être penser à une publication numérique... à un traitement dynamique... et à l'image de ton film... Nous ne voudrions pas non plus que ce soit trop bavard. Cela ne sera pas une histoire du théâtre mais cela va y contribuer, parce que cela reste encore à faire, parce qu'il y a la liaison avec la vie théâtrale à Brazzaville, nous allons en parler évidemment...

JP : Mais cela ne va pas être systématique.

NMG : En ce moment, nous nous centrons vraiment sur l'acquisition du texte. Nous nous confrontons à de nombreuses questions techniques. Nous nous sommes réparti les tâches bien sûr, mais il y a des tapuscrits de travail que nous avons récupérés à 
Brazzaville, ils sont tout poisseux de la sueur des répétitions. Comment rendre cela par des notes? Que faire des traces de la mise en scène?

JP : C'est ça qui est intéressant à l'intérieur des textes qui nous ont été remis. Souvent on recueille des documents de ce genre: un comédien de Sony qui a écrit des indications de mise en scène dans les tapuscrits de travail et qui nous remet ces tapuscrits de répétition... Clément Batsimba par exemple dont je parlais tout à l'heure. Maintenant il faut connaître le mode d'emploi... Ils ont changé le nom du personnage... Il y a les notes de comédiens, de ce qui est en train de se jouer au plateau...

Fig. 7 : Tapuscrit de travail de Clément Batsimba dit Ya Bat, comédien du Rocado Zulu Théâtre, en répétition de Qui a mangé Madame Davoine Berghota, avec le metteur en scène Jean-Pierre Klein (Brazzaville, août-septembre 1989)
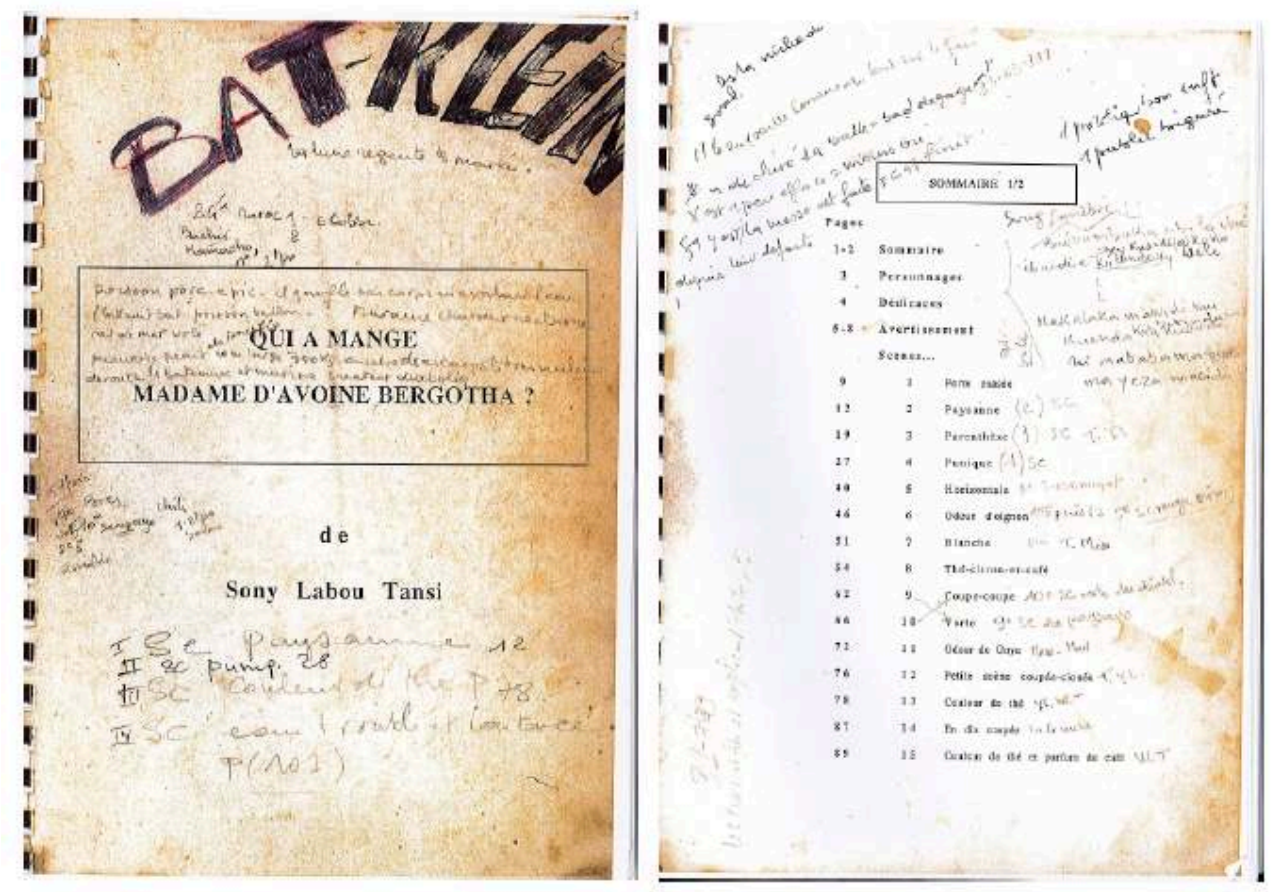

NMG : On ne peut pas publier tout cela...

JP : Mais cela peut s'analyser, c'est ce qu'on fait pour chaque pièce.

NMG : On ne va pas faire une histoire générale du théâtre à Brazzaville...

\section{$A D$ : Cela apporte nécessairement des jalons...}

JP : C'est le but parce que quand tu travailles le théâtre, tu ne peux pas le travailler en dehors de cette question, ce n'est pas quelque chose à part, ce n'est pas quelque chose qui est détaché d'un contexte, tous ces éléments sont nécessairement intriqués. C'est ce qui fait le théâtre de Sony, cela n'aurait aucun sens pour nous de le détacher de tous ces éléments de génétique élargie. Si l'on faisait un travail uniquement sur la transformation du texte comme dans les poèmes, on ne serait pas dans le théâtre de Sony, qui est un théâtre qui s'est façonné dans l'échange, dans le contact, dans les mises en scène...

NMG : C'est ce qu'il dit dans la dernière archive qu'on a trouvée, qui provient d'Émile Lansman $^{27}$, son éditeur. Cela fait partie du travail que nous n'avons pas fini. 
JP : Ce travail avec Lansman, on ne l'a pas encore fait, on espère le faire. Ainsi, nous avons encore des pistes à explorer.

NMG : On est un peu débordé...

JP : On n'a pas de limites dans le théâtre, cela vient de l'objet théâtre. [...]

Fig. 8 : Page d'agenda (année inconnue), notes inédites sauf « Mon écriture... », publiée dans L'autre monde $^{28}$

(C) Fonds Sony Labou Tansi de la Bibliothèque francophone multimédia de Limoges²9

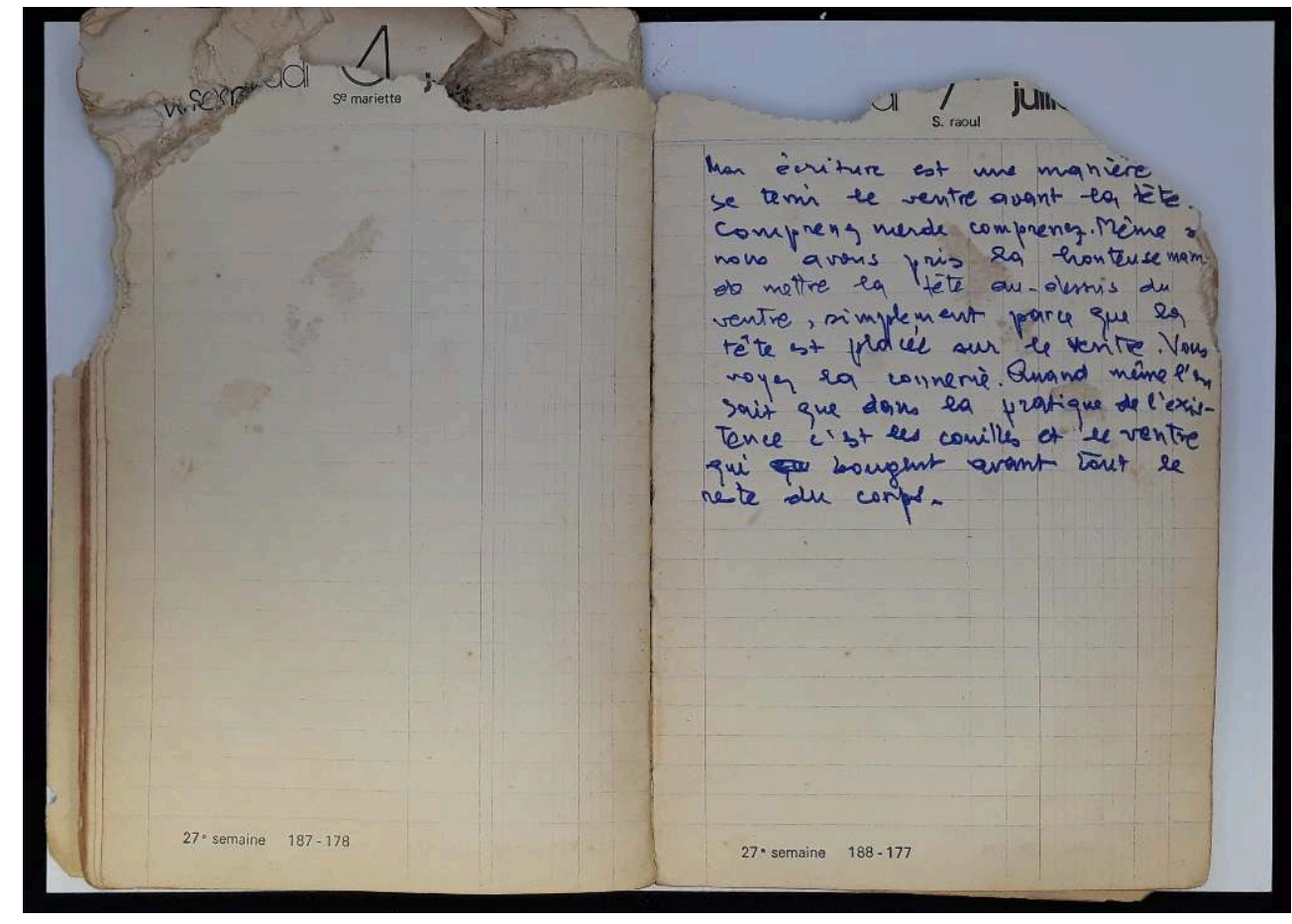

Fig. 9 : Pages d'agenda (1980), notes sur l'acteur (recto et verso)

(C) Fonds Sony Labou Tansi de la Bibliothèque francophone multimédia de Limoges ${ }^{30}$ 

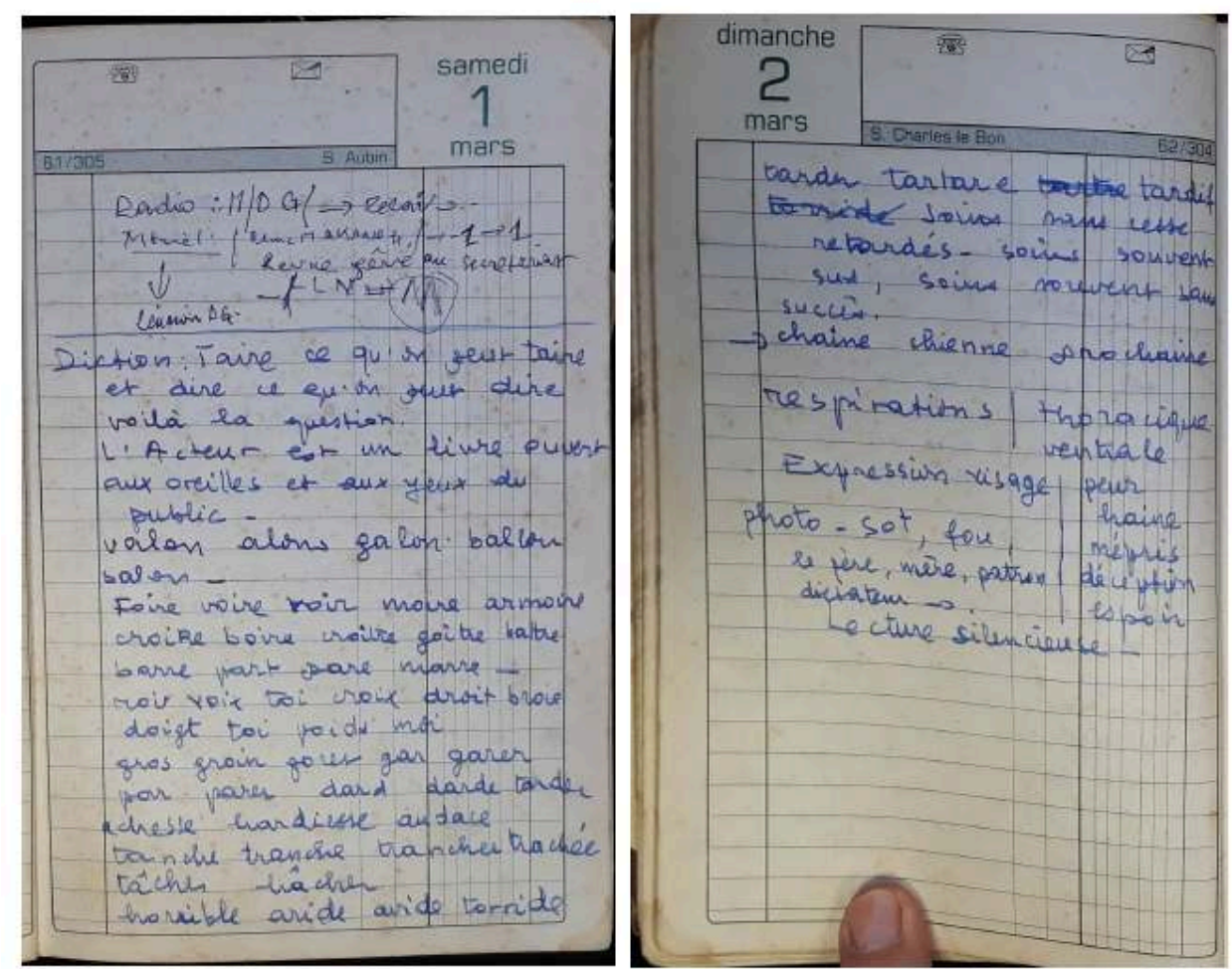

JP : Les agendas sont par exemple aussi un outil de travail, c'est-à-dire que dans ses agendas, on en a très peu - on vient d'en retrouver un dans une cantine d'archives gardée par sa fille Darmala à Brazzaville -, il y a la matérialité de ses rendez-vous, de ses rencontres, de ses contacts, et en même temps l'écriture qu'il met en parallèle. Darmala nous a ouvert, dans le jardin de sa maison, l'accès à ses deux cantines. Nous y sommes allés avec le cousin de Sony, Victor Mbila Passi, et en photographiant, nous avons retrouvé des documents à la fois très intimes et sur la politique qu'on n'a pas pu récupérer, parce que c'est un aspect de la vie de Sony quand il était député qui ne nous concerne pas, mais aussi une correspondance avec Tchicaya U Tam'si et un agenda qui n'a pas été archivé. Ce sont des documents extrêmement précieux pour notre travail sur le théâtre. Comme une manière de revisiter une cantine à l'aune de chaque recherche et de recréer des archives différemment...

$A D$ : Votre travail débouche aussi sur la volonté de laisser des traces créatives: de perpétuer cette archive mais à un niveau artistique. On peut penser à ce que vous avez fait au sein de l'équipe autour de la bombe Sony ${ }^{31}$, mais également au film documentaire que tu as réalisé sur Sony : Je ne suis pas vivant mais poète ${ }^{32}$ dans sa version courte, Insurrection du verbe aimer dans sa version longue. Est-ce que tu peux revenir sur cette expérience?

JP : Mon film est un travail extra-universitaire, qui est devenu une nécessité personnelle après mon expérience au Conservatoire. Je me suis investie dans un atelier documentaire de neuf mois à la Fémis en 2014 et j'ai commencé à filmer à Brazzaville cette même année. Au point de départ de ma quête de ce qui reste aujourd'hui de cette parole poétique de Sony Labou Tansi, se déploient les trajectoires de trois hommes de théâtre : Dieudonné Niangouna, Étienne Minoungou et Jean-Paul Delore. Avec eux, se dessinent trois visages contemporains et rêvés de Sony Labou Tansi. Il y a celui incarné par Dieudonné Niangouna, son fils spirituel, qui vit désormais cette relation depuis la France, car il est menacé dans son pays depuis octobre 2015, du fait de son opposition au changement de la Constitution de Denis 
Sassou Nguesso, dictateur en place ; celui porté par Étienne Minoungou, qui trouve dans les écrits de Sony le terreau de sa lutte et de ses engagements par l'art et le théâtre depuis le Burkina Faso, où il a lutté avec tout le mouvement du Balai citoyen ; et celui figuré par Jean-Paul Delore, dépositaire lui aussi de la parole de Sony, par le choix de ses rencontres et collaborations et ses multiples allers retours artistiques entre l'Europe et l'Afrique. Avec les festivals qu'ils organisent à Brazzaville - le Mantsina-sur-scène, où une édition entière a été consacrée à Sony en 2015 par son directeur artistique pendant douze ans, Dieudonné Niangouna - et à Ouagadougou les Récréâtrales-, leur rôle de passeurs pour différents publics et générations m'a particulièrement inspirée. Un quatrième passeur s'est imposé au fur et à mesure du tournage, Zora Snake, performeur camerounais, qui a comme «incorporé » Sony dans son art et dont la poésie des performances m'a permis de mieux saisir ce que Sony veut exprimer quand il dit que les mots sont " des bulles de silence qui parlent " ou « des cadavres qui aspirent à la résurrection ». J'ai tissé mon film avec des textes de Sony (ses poèmes en particulier, une pièce montée par Dieudonné Niangouna à la Colline, «Antoine m'a vendu son destin/ Sony chez les chiens » ou encore des textes à portée plus philosophique ou des inédits comme un extrait de « Le Mort te dit adieu toi qui restes vivant ») pris à bras le corps par ces artistes et beaucoup d'autres, dans les rues ou sur les plateaux, entre Brazzaville, Ouagadougou et Paris.

\section{NOTES}

1. Nicolas Martin-Granel, «Sony Labou Tansi, afflux des écrits et flux de l'écriture », Continents Manuscrits, $\mathrm{n}^{\circ} 1,2014$.

2. Labou Tansi, Sony, L'Atelier de Sony Labou Tansi. Nicolas Martin-Granel et Greta RodriguezAntoniotti (éd.), vol. I. Correspondance ; vol. II. L'Acte de respirer, suivi de 930 Mots dans un aquarium (poésie) ; vol. III. Machin la Hernie (roman), Paris, Éditions Revue Noire, 2005, 264 p. + 216 p. + 304 p. (présenté dans un coffret).

3. Sony Labou Tansi, Encre, sueur, salive et sang, Greta Rodriguez Antoniotti (éd.), Paris, Le Seuil, 2015.

4. Nicolas Martin-Granel, Claire Riffard (dir.), en collaboration avec Céline Gahungu, Sony Labou Tansi, Poèmes, édition critique et génétique de l'œuvre poétique, Paris, CNRS Éditions, 2015.

5. Nicolas Martin-Granel, Julie Peghini (dir.), Sony Labou Tansi en scène(s), La Chair et l'Idée, théâtre et poèmes inédits, lettres, témoignages, écrits et regards critiques, Besançon, Les solitaires intempestifs, 2015.

6. Ibid.

7. Responsable du concours théâtral interafricain.

8. Avec la collaboration artistique de Julie Peghini, notamment pour le choix des textes.

9. Fondé à Brazzaville en 2003 par un collectif de jeunes dramaturges et comédiens : Dieudonné Niangouna, Abdon Fortuné Koumbha, Ludovic Louppé, Jean Félhyt Kimbirima et Arthur Batouméni. Cf. https://festivalmantsina.wordpress.com. Pour une petite histoire du festival à l'occasion des dix ans de Mantsina, aux Francophonies en Limousin, voir aussi l'archive vidéo de la table ronde «Fenêtre ouverte sur le festival Mantsina sur scène: dix ans de résistance 
théâtrale !»: http://www.theatre-video.net/video/Festival-Mantsina-10-ans-de-resistancetheatrale-31e-Francophonies-en-Limousin.

10. http://www.item.ens.fr/groupe-sony-labou-tansi/

11. Association pour l'étude des littératures africaines

12. Nicolas Martin-Granel fait ici référence au stage de direction d'acteurs par Jean-Damien Barbin et Dieudonné Niangouna au Conservatoire national supérieur d'art dramatique en 2013.

13. Nom familier donné à l'auteur-metteur en scène et comédien Dieudonné Niangouna.

14. Nicolas Martin-Granel, «La quadrature du texte ou l'énigme des quatre "Le quatrième côté du triangle" de Sony Labou Tansi », Genesis, n 33, 2011, p. 53-66.

15. Équateur, $n^{\circ} 1$, dirigé par Caya Makhélé en 1985 à Paris. Jean-Damien Barbin a conservé un exemplaire reçu à Nantes lorsqu'il accueille la tournée d'Antoine m'a vendu son destin, mis en scène par Daniel Mesguich.

16. Julie Peghini, Xavier Garnier (dir.), Le Théâtre de Sony Labou Tansi, $\mathrm{n}^{\circ} 41$, Études littéraires africaines, 2016.

17. Directrice du Festival des Francophonies en Limousin de 1984 à 2000.

18. Gabriel Garran, metteur en scène de Je soussigné cardiaque en 1985. Il a été le fondateur du Théâtre de la Commune en 1971 et du TILF (Théâtre international de langue française) en 1993.

19. Jean-Marc Devésa est professeur des universités à Limoges, spécialiste des littératures francophones extra-européennes. Auteur de Sony Labou Tansi. Écrivain de la honte et des rives magiques du Kongo, Paris, L'Harmattan, 1996, 379 p.

20. Karthala, 2015. Voir aussi Martin-Granel, Nicolas : " Une poétique de la contagion », in « Sony Labou Tansi à l'œuvre ", Itinéraires et Contacts de Cultures, vol. 40 , L'Harmattan, 2007, p. 145-159.

21. Association nationale des écrivains au Congo.

22. Le titre même est incertain, certains témoins l'appellent Franco loge Dieu.

23. Allusion à la Conférence nationale souveraine, le grand événement politique contemporain.

24. Co-fondateur du Rocado Zulu Théâtre.

25. Sony consacre une pièce de théâtre à Kimpa Vita, dont il existe deux mentions bibliographiques, « Béatrice du Congo » (1980) et Simpa Kimpa Mvita (1981). Le 29 octobre 1665, les troupes portugaises basées à Luanda mettent fin au règne du manikongo Vita Nkanga (Antonio $\mathrm{I}^{\mathrm{er}}$ ) dont l'armée est défaite à Mbwila. Engagé dans cette guerre pour arrêter les saignées esclavagistes qui ruinaient son pays, le roi défait est décapité. Le royaume déjà affaibli par les divisions provoquées par la traite s'effondre, en proie à une longue et interminable guerre civile. C'est dans ce contexte qu'en 1706 apparaît une jeune fille d'une vingtaine d'années Dona Béatrice, Kimpa Vita, qui se revendique de saint Antoine et qui se charge de réhabiliter la grandeur du Kongo. Sur Kimpa Vita, voir Georges Balandier, Le Royaume de Kongo du XVI au XVIII siècle, Paris, Fayard/Pluriel, 2016, et tout récemment Abel Kouvouama, Une histoire du messianisme. Un « monde renversé », Paris, Karthala, 2018.

26. Sony consacre une pièce de théâtre au grand chanteur Franco, Franco, loge de Dieu. Pièce de théâtre musicale dont ne reste aucune trace écrite, créée par Sony Labou Tansi et le Rocado Zulu en 1989 en hommage au grand chanteur zaïrois François Luambo Luanzo Makiadi, connu sous les noms de Franco Luambo ou, plus simplement, de Franco. Selon la tradition orale le titre est variable : parfois Franco, l'âge de Dieu, le plus souvent Franco, loge de Dieu.

27. Lire le témoignage qu'il donne de sa première rencontre avec Sony et la découverte de l'état chaotique des manuscrits que le dramaturge le presse d'éditer en urgence. «Et Sony me répond : “tu m'emmerdes, Émile, qu'est-ce que tu veux, être éditeur ou imprimeur? Si tu veux être imprimeur, ça ne m'intéresse pas. Je sais bien que j'ai des défauts dans mon écriture, j'ai besoin de quelqu'un pour me botter le cul.” » («En compagnie d'Émile », Le Matricule des Anges, n²02, avril 2019, p. 15). 
28. Sony Labou Tansi, L'Autre monde. Écrits inédits. Choix des textes réalisé par Nicolas MartinGranel et Bruno Tilliette à partir des nombreux cahiers et notes manuscrites, Paris, Éditions Revue noire, 1997, p. 77.

29. http://sonylaboutansi.bm-limoges.fr/

30. http://sonylaboutansi.bm-limoges.fr/

31. http://eman-archives.org/francophone/exhibits/show/sony-ou-la-bombe-h/la-bombe-hsony

32. Diffusé sur TV5 monde en février 2019.

INDEX

Mots-clés : Sony Labou Tansi, archives, théâtre

Keywords : Sony Labou Tansi, archives, theater 